\title{
Chest wall reconstruction with digitally designed materials for straight back syndrome with tracheal stenosis: a case report
}

\author{
Yang Liu, Wenlin Wang, Weiguang Long, Bin Cai, Chunmei Chen, Wenjie Wang, Shubin Guan, \\ Juan Luo, Kai Chen \\ Department of Chest Wall Surgery, Guangdong Second Provincial People's Hospital, Guangzhou, China \\ Correspondence to: Wenlin Wang, MD. Department of Chest Wall Surgery, Guangdong Second Provincial People's Hospital, Guangzhou 510317, \\ China. Email: willinew@126.com.
}

\begin{abstract}
Severe symptoms of straight back syndrome (SBS) occur through compression of the mediastinal structures. The treatment is to relieve compression, but surgical relief has not been reported. A 29-year-old woman was admitted to hospital because of dyspnea for 2 years. Physical examination showed the physiological curvature of the spine had disappeared and imaging examination confirmed that the spine was straight, the distance between the spine and the anterior chest wall was shortened, and the lower part of the trachea was obviously compressed with a severe stenosis. The chest wall was simulated by $3 \mathrm{D}$ technology, and a model was obtained by $3 \mathrm{D}$ printing. The surgical resection was simulated on the model, and the required replacement material was digitally designed and processed. The upper half of the sternum, the first and second costal cartilages, and the thymus were excised. After the chest wall defect was repaired with the digital material, the ascending aorta was suspended on the reconstruction to relieve the tracheal compression. The operation was completed in 135 min without any complications. All symptoms disappeared after operation. Postoperative imaging examination showed complete resolution of the tracheal stenosis. The patient was discharged 10 days after operation. Surgical reconstruction of the anterior chest wall is a good method for treating tracheal stenosis in SBS, and digitally produced material is ideal for the reconstruction.
\end{abstract}

Keywords: Chest wall reconstruction; digital materials; straight back syndrome (SBS); tracheal stenosis; case report

Submitted Jun 17, 2021. Accepted for publication Aug 20, 2021.

doi: 10.21037/atm-21-3976

View this article at: https://dx.doi.org/10.21037/atm-21-3976

\section{Introduction}

Straight back syndrome (SBS) is an autosomal dominant disorder, first reported by Rawlings in 1960 (1,2). It is mainly characterized by the disappearance of the physiological curvature of the spine, straightness of the upper thoracic vertebra, shortening of the anteroposterior diameter of the thorax, and compression of the structures in the mediastinum, resulting in variety of symptoms (3-6). The fundamental cause of the disease is spinal, and the symptoms are mainly concentrated on the respiratory and cardiovascular systems. SBS is a congenital disease and spinal lesions are present at birth. Most patients have mild symptoms that manifest as simple cardiac or respiratory discomfort and can be relieved with medication. A minority of patients are more symptomatic and require special treatment, which is usually given symptomatic treatment such as prevention of infection, oxygen inhalation, resolving phlegm, relieving asthma and ventilator-assisted breathing. There are also treatment methods of stent implantation under fiberoptic bronchoscope to dilate narrow bronchus. There is no previous reports of SBS operation with our method in a thoracic surgery department $(7,8)$. We recently treated a patient with SBS who had a severe stenosis of the trachea due to external compression, presenting with severe respiratory tract obstruction. Because conservative treatment was not effective, we proceeded with a surgical treatment. We present the following article in accordance with the CARE reporting checklist (available at https:// 

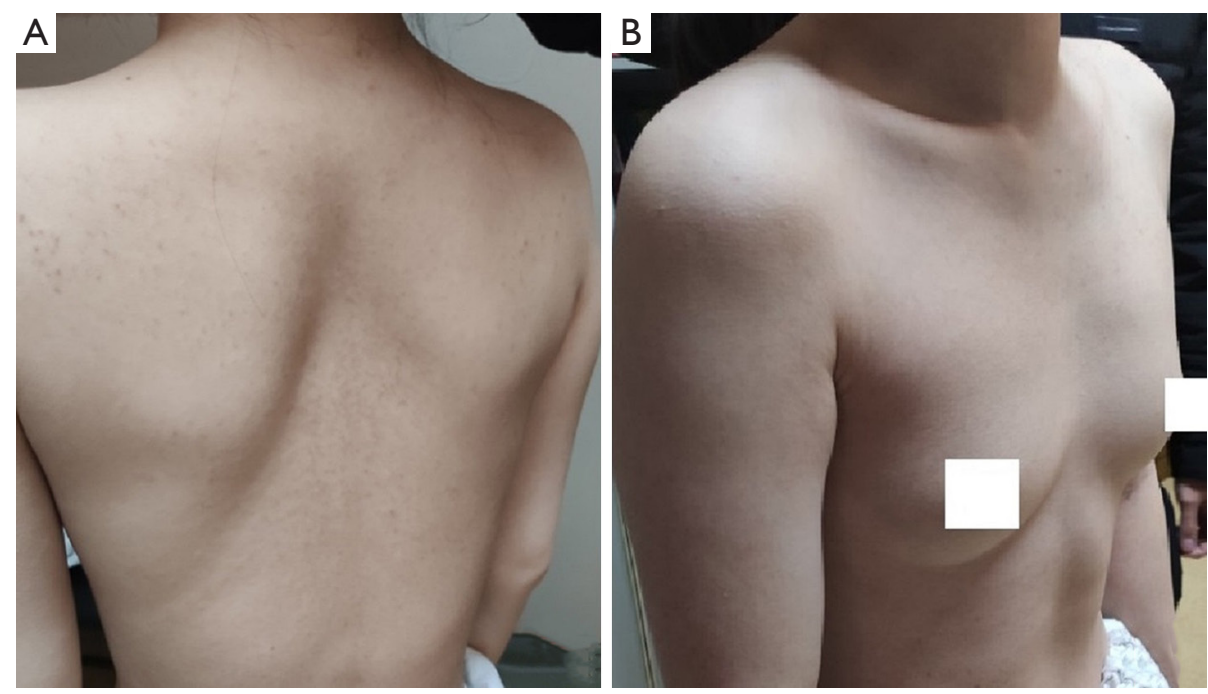

Figure 1 Patient appearance. Appearance of (A) the back and (B) the anterior chest wall of the SBS patient. No physiological curvature of thoracic spine was seen. This image is published with the patient's consent.

dx.doi.org/10.21037/atm-21-3976).

\section{Case presentation}

\section{Clinical background}

A 29-year-old woman was admitted to hospital because of dyspnea for 2 years. Before admission, she had severe dyspnea of unknown cause. Myasthenia gravis had been suspected at a local hospital, but the diagnosis was excluded after examination. She was finally diagnosed as SBS. As her symptoms continued to worsen, she attended The First Affiliated Hospital of Guangzhou Medical University 4 months before the current admission. Bronchiectasis test results suggested severe restrictive pulmonary ventilation dysfunction. On examination, her bronchus was found to be obviously compressed and tracheal stent implantation was planned, but failed due to severe tracheal stenosis during the operation. After treatment with prednisolone, levofloxacin, compound methoxyphenamine, ambroxol and other drugs, her symptoms were relieved, but they recurred after drug withdrawal and became more severe. The final diagnosis was SBS with severe tracheal stenosis. She was referred to the Department of Chest Wall Surgery, Guangdong Second Provincial People's Hospital for further treatment.

On admission she had dyspnea, cough, expectoration, fatigue and occasional dizziness. Her oxygen saturation is $\sim 92 \%$ without oxygen support, and with oxygen support, it was maintained at $96-99 \%$. Physical examination showed that the appearance and shape of the anterior chest are essentially normal, without depression or a flat chest, the spine was straight without the physiological curvature, and the thoracic spine was slightly convex forward (Figure 1). The heart beat was visible on the left anterior chest wall, and a grade II systolic murmur was heard in the second intercostal space to the left of the sternum. The results of computed tomography (CT) and chest threedimensional (3D) imaging examinations showed that the thorax appeared normal, the physiological curvature of the spine had disappeared, the thoracic spine was straight, the distance between the anterior chest wall and the thoracic spine was obviously reduced, the thymus was enlarged, the aorta was slightly thicker, and the trachea was obviously compressed, with only a narrow passage in the lower segment, and the most severe compression located at the beginning of the aortic arch (Figures 2,3). Pulmonary function examination showed severe restrictive pulmonary ventilation dysfunction. Cardiac ultrasound examination indicated that the diameter of the ascending aorta was $31 \mathrm{~mm}$, the trunk of the pulmonary artery was $19 \mathrm{~mm}$, the left atrium was $21 \mathrm{~mm}$, the right atrium was $19 \mathrm{~mm}$, diastolic right ventricular was $24 \mathrm{~mm}$, diastolic left ventricular was $36 \mathrm{~mm}$ and the systolic left ventricular was $22 \mathrm{~mm}$. Based on all the clinical data, the preoperative diagnosis was SBS with severe tracheal stenosis. She had indications and no contraindications for surgical treatment.

Thyroid nodules had been found 2 years ago and 

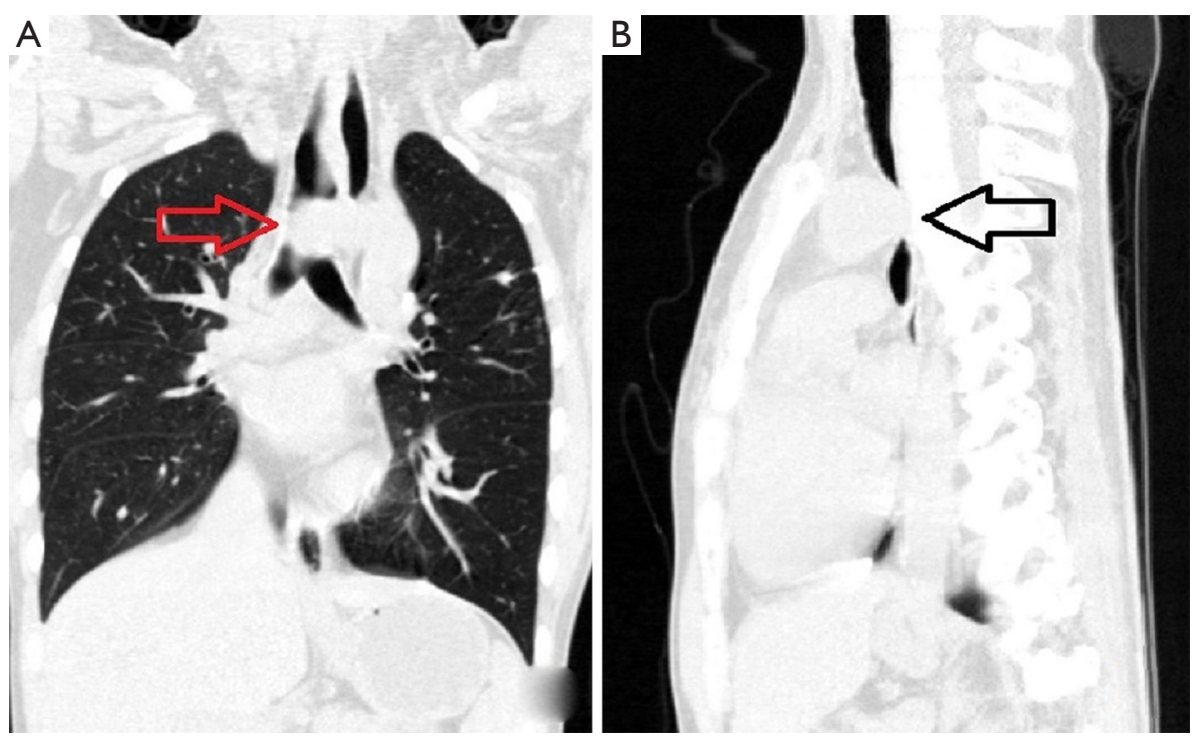

Figure 2 Computer tomography examination show compression of the trachea by the beginning of the aortic arch. (A) Aortic arch compressing the trachea to cause stenosis (red arrow); (B) the trachea is severely compressed (white arrow).
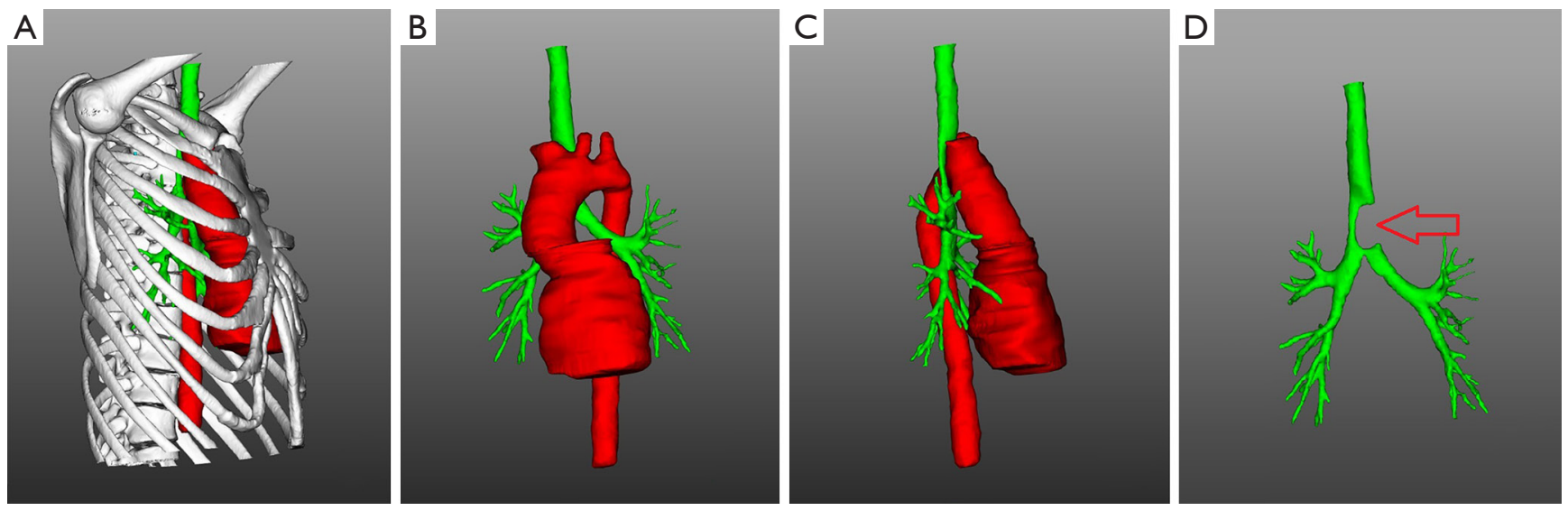

Figure 3 Preoperative three-dimensional reconstruction image of the chest. (A) Straight spine, shortened distance to anterior chest wall, obvious compression of mediastinal structures; (B) relationship between ascending aorta, aortic arch and trachea; (C) obvious compression of the trachea resulting in (D) a defect of the tracheal image (red arrow).

radiofrequency ablation was performed in a local hospital. Tuberculosis was diagnosed in 2017, and cured after 18 months of standard treatment. The patient had no special family or psycho-social history.

She and her family members expressed their understanding and consent to the treatment plan, and signed the consent forms related to the operation. All procedures performed in studies involving human participants were in accordance with the ethical standards of the institutional and/or national research committee(s) and with the Helsinki Declaration (as revised in 2013). Written informed consent was obtained from the patient for publication of this case report and accompanying images. A copy of the written consent is available for review by the editorial office of this journal.

\section{Diagnostic standard}

(I) Physical examination showed changes of straight back flat chest or funnel chest, and murmur could be heard at 

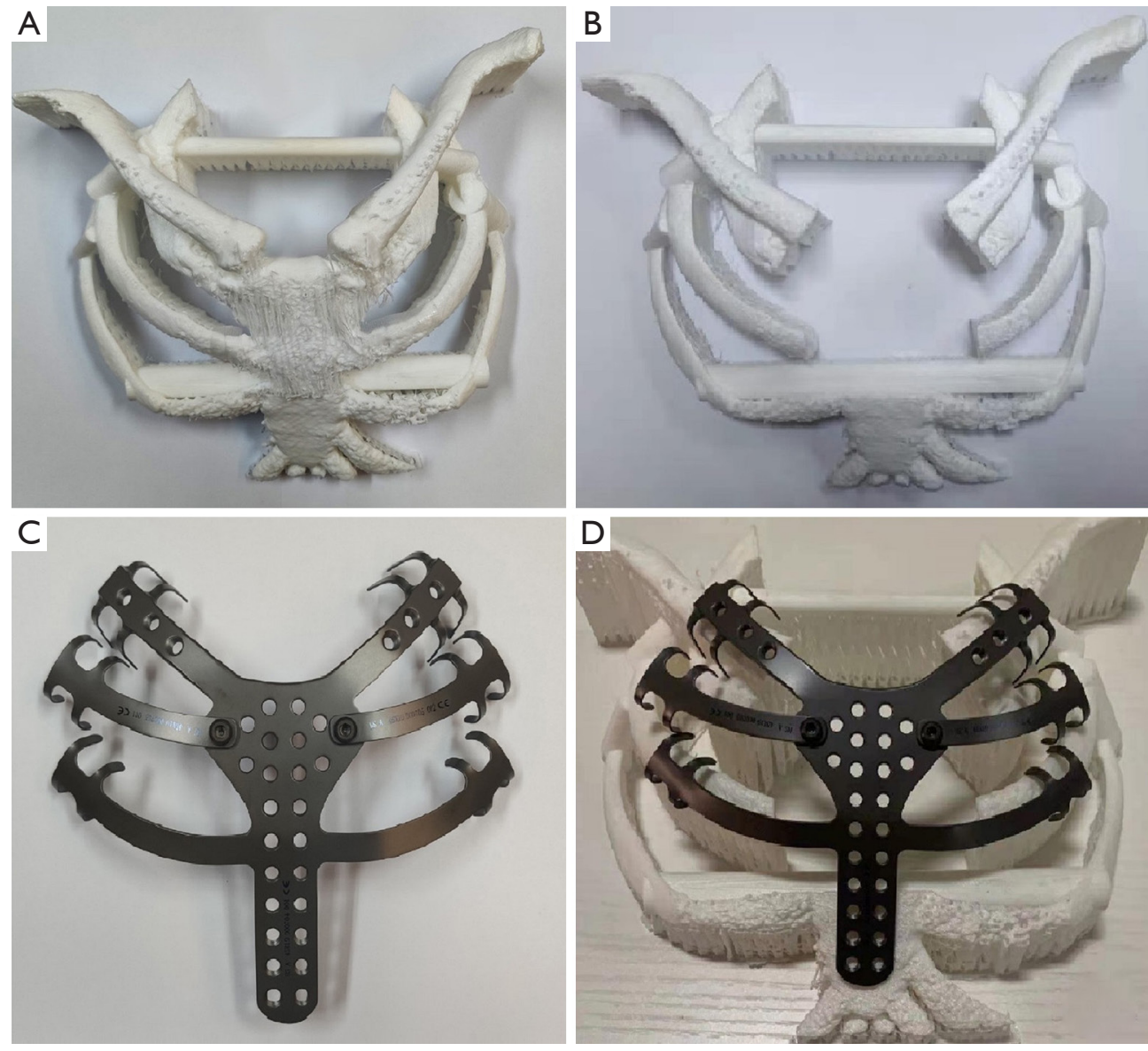

Figure 4 Design and production of digital materials. (A) Making a thoracic model by 3D printing; (B) simulating the resection of bony structure on the model; $(\mathrm{C})$ processing the digital materials according to the range of the resection; (D) simulating the placement of the digital materials during operation.

the bottom of the heart; (II) in lateral chest radiograph, the vertical distance between the front edge of T8 vertebral body and T4-T12 line is less than $1.2 \mathrm{~cm}$; (III) chest anterior posterior diameter/transverse diameter ratio $\leq 0.33$; (IV) organic heart disease was excluded.

\section{Digital production of reconstruction materials}

The patient's chest was scanned with enhanced CT to obtain DICOM data that was used to reconstruct the chest with 3D technology. A model was created with 3D printing technology for simulation of the surgical resection, designing the basic shape of the reconstruction material according to the surgical need, and finally producing the required materials according to the design (Figure 4).

\section{Surgical method}

Following standard preoperative preparation, general anesthesia was induced. A schematic diagram of the operation is shown in Figure 5. With the patient supine, a $10-\mathrm{cm}$ median incision of the anterior chest wall was made. Separation of the subcutaneous and muscle tissues bilaterally revealed the bony structure of the anterior chest wall. The upper sternum and the left and right first and second costal cartilages were removed. During the operation, the enlarged thymus gland was excised (Figure 6A), to reveal the ascending aorta, aortic arch and heart, which were attached to the sternum (Figure $6 B$ ). The pericardium was cut at the pericardial fold, and the ascending aorta was passed through the traction line of a sling (Figure 6C), which was then pulled by the traction line 
A

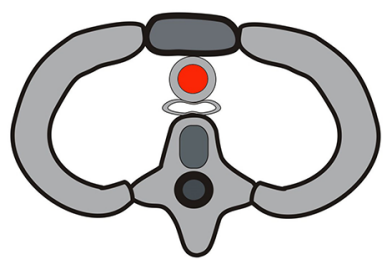

C

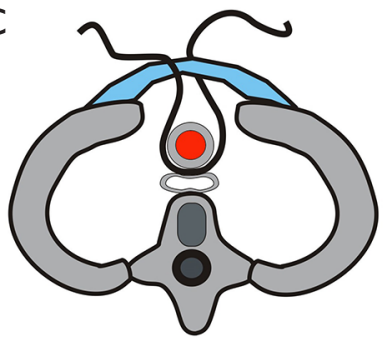

B

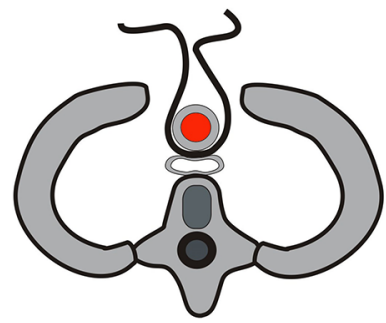

$\mathrm{D}$

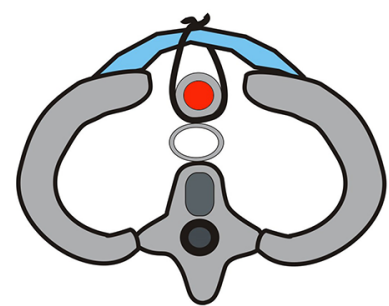

Figure 5 Schematic diagram of the operation. (A) The aorta and trachea are located between the thoracic vertebrae and the anterior chest wall, and the trachea is obviously compressed; (B) removal of part of the anterior chest wall, and placement of a sling around the ascending aorta; (C) reconstruction of the anterior chest wall with the digital materials; (D) tightening of the sling to raise the ascending aorta and eliminate tracheal compression.
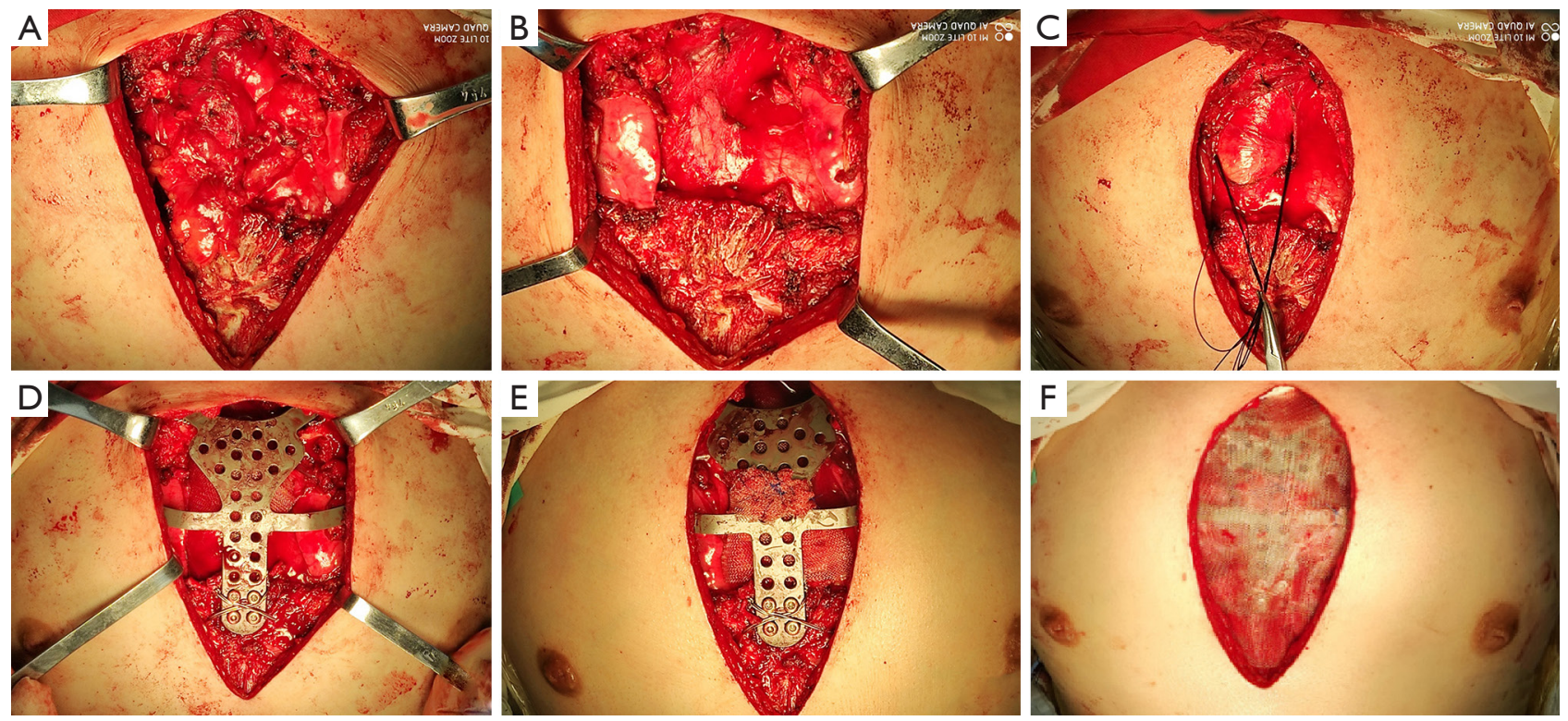

Figure 6 Images of the operation. (A) Excision of the upper half of the sternum and the first and second costal cartilages to expose the upper mediastinum; (B) excision of the thymus to expose the ascending aorta and aortic arch; (C) placement of a sling around the ascending aorta; (D) reconstruction of the anterior chest wall with the digital materials; (E) tightening of the sling to raise the ascending aorta and fixing the sling on the surface of the digital materials; (F) covering the digital materials with a fibrous membrane, closing the incision. 

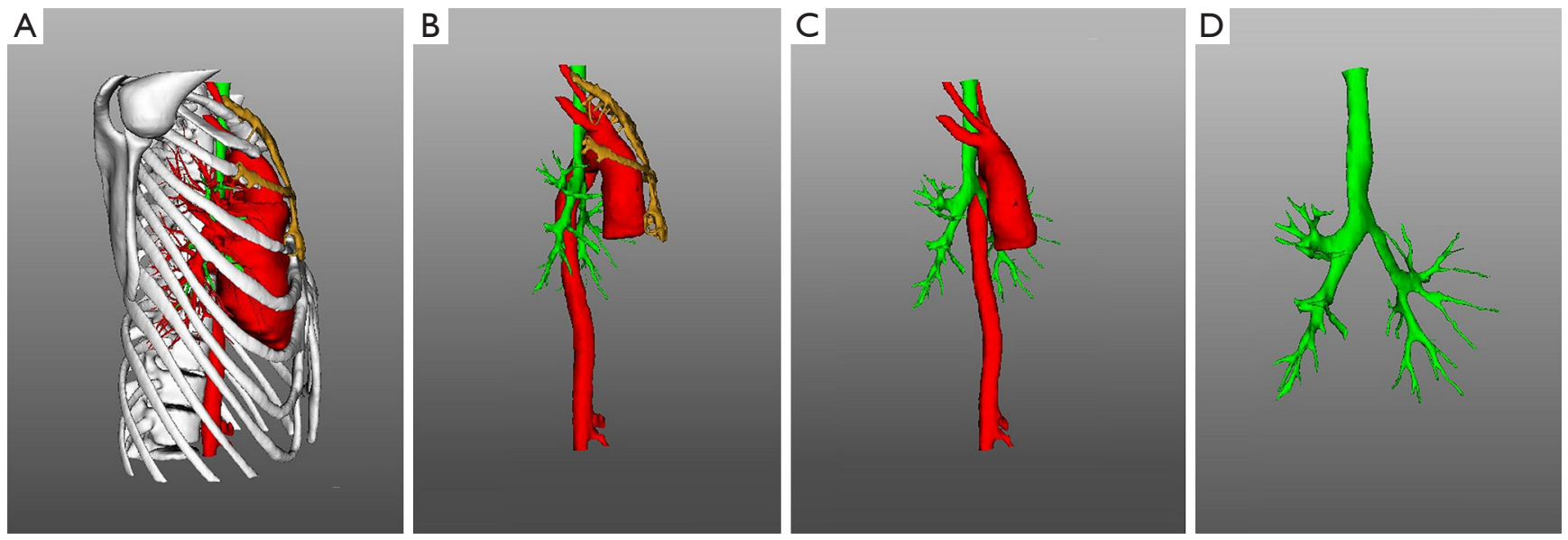

Figure 7 Three-dimensional reconstruction image after thoracic surgery. (A) The distance between the anterior chest wall and the spine has significantly widened; (B) there is a gap between the ascending aorta and the trachea is no longer compressed; (C) spatial relationship between the ascending aorta and the trachea; (D) elimination of tracheal compression and the normal caliber is restored.

to pass the ascending aorta. The anterior chest wall was reconstructed with the digital material, which was placed on the outer surface of the bony structure (Figure $6 D$ ). The distance between the material and the anterior wall of the ascending aorta was $\sim 2 \mathrm{~cm}$, so we tightened the sling around ascending aorta to advance it by $1.5 \mathrm{~cm}$ (Figure $6 E$ ). After covering the digital material with a fibrous membrane (Figure 6F), placing drainage tubes in the bilateral thoracic cavity and surgical field, closing the incision layer by layer, the operation was completed.

\section{Outcomes}

After the ascending aorta was suspended during the operation, the tracheal trunk was probed manually, and there was no longer any compression. The airway pressure was $23 \mathrm{cmH}_{2} \mathrm{O}$ before ascending aorta suspension, and immediately decreased to $12 \mathrm{cmH}_{2} \mathrm{O}$ after the suspension. The operation lasted $135 \mathrm{~min}$, and blood loss was $50 \mathrm{~mL}$. The operation went smoothly without any complications. The patient recovered uneventfully and the symptoms of dyspnea were significantly improved. The cough and expectoration gradually improved with symptomatic treatment. The bilateral thoracic drainage tubes were removed 3 days after operation, and the surgical field drainage tubes was removed 6 days after operation. Reexamination of CT and 3D images showed a clear distance between the ascending aorta and trachea. The trachea essentially regained its normal caliber, and the stenosis disappeared (Figure 7). Biopsy revealed hyperplasia of the thymus. The patient was discharged 10 days after operation. Her oxygen saturation was normal without oxygen therapy, and no obvious murmur was heard in each valve of the heart (Figure 8).

\section{Discussion}

The spine is the main load-bearing structure of the human body. In the process of human evolution, in order to adapt to upright walking, the spine gradually evolved a physiological curvature. Under normal development, human thoracic vertebrae form a backward bend (9), which buffers the organs during exercise, and provides enough space to accommodate the thoracic organs. In SBS patients, the curvature of the thoracic spine is replaced by a straight spine. The straight thoracic spine narrows the distance to the anterior chest wall, which reduces the mediastinal space, leading to compression of the heart, great vessels, esophagus, trachea etc. (1-5).

Due to the uneven distribution of the structures in mediastinum, each shows different effects of being compressed. The heart is the largest structure, and is most vulnerable to compression. The right ventricular outflow tract is located adjacent to the sternum, and is the first site of compression. There are various symptoms when the heart is compressed (3-5), and many SBS patients are initially diagnosed for heart problems, so SBS is also called false heart disease (3). The aorta is a structure with continuous 


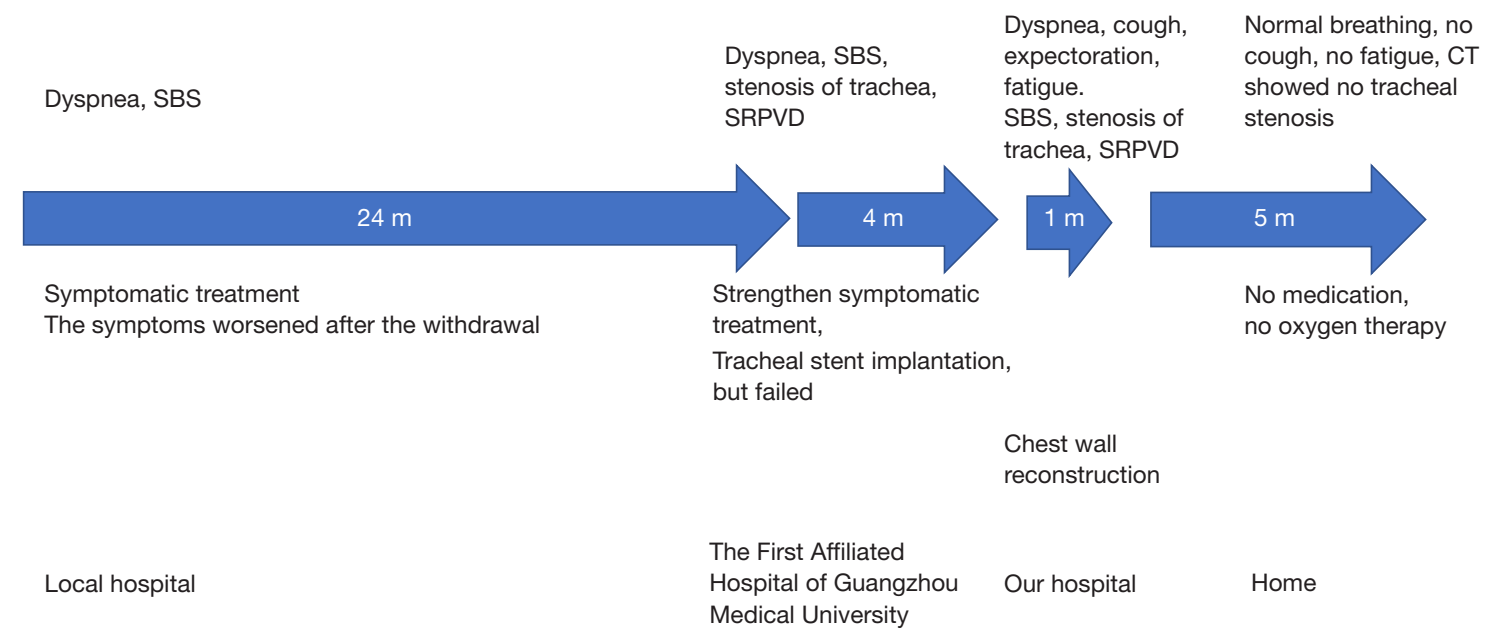

Figure 8 Time line of clinical history, including the diagnosis, symptoms, treatment methods, and objective response to the results of treatment.

positive pressure, and strong pressure resistance, so it is not easy to change its shape under external pressure. However, that external pressure may be transmitted to surrounding structures, causing them to be compressed. The trachea descends along the midline in front of the spine, and the beginning of the aortic arch crosses over the lower segment of the trachea. Under normal circumstances, the mediastinal space is large enough that the aorta will not significantly compress the trachea. However, when the mediastinal space becomes smaller, the compression of the anterior chest wall is transmitted to the trachea through the ascending aorta. Because the lower end of trachea divides into the left and right bronchi, the two bronchi have a strong pulling effect, which prevents the trachea from sliding to one side of the vertebral body when it is pressed forward, and thus the airway collapses and narrows. Breathing disorders occur when the constriction is severe. The esophagus is also an important structure in the mediastinum, but it mainly runs along the left side of the spine, where it is protected from compression and blockage by the anterior edges of the vertebral bodies. Therefore, among the various structures in the mediastinum, the heart and trachea are the two that are easiest to compress, and related symptoms are the most likely to appear.

The basic cause of SBS is the reduction of space between the spine and anterior chest wall. Theoretically, there are two ways to widen this space: bend the straight spine backward to form a normal physiological curvature or make expand the chest wall. The spine is extremely difficult to bend backwards. External traction has been used to perform correction, but it is a huge undertaking for the patient and the effect is not ideal $(5,6)$. It seems even more unrealistic to perform direct surgical correction of the spine. The shape of the anterior chest wall in SBS patients is generally normal, but because the distance between the spine and the anterior chest wall cannot be effectively widened by existing methods, only conservative methods have been used to treat patients clinically $(7,8)$.

This patient had severe patient, and could not perform normal activities of daily living because of the severe tracheal compression. Placement of a tracheal stent had been the last hope of conservative treatments, but it failed. Therefore, the only treatment remaining was an operation on the anterior chest wall.

Essentially, there are two basic ways to expand the chest wall shape: shaping (10-12) or reconstruction (13). The former changes the shape without damaging the original chest wall structure, while the latter completely changes the shape of the chest wall. Shaping is more suitable for minimally invasive surgery, whereas reconstruction is mostly open surgery. The aim of the operation is to make the SBS patient's anterior chest wall convex. In theory, minimally invasive treatment is ideal, but at present, all mature minimally invasive operations are performed on deformed structures (10-12). The anterior chest wall structure of SBS patients is completely normal, and there is no deformity. In order to expand chest wall forward, the popular minimally invasive procedures are ineffective (10-12). Therefore, 
to achieve the purpose of treatment, we have to do a reconstruction; that is, open surgery.

The general idea is that if the chest wall needs to be more convex anteriorly, it can be softened completely, manipulated to the front, and then fixed as needed. Although such an idea is possible, it is unrealistic. Because of the limited circumference of the thorax, it is obviously difficult to move the chest wall with limited length forward. After detailed research, we found that a reasonable method is to remove the existing chest wall structure, and use an artificial material to reconstruct it to the required convexity, which can achieve more easily.

There are three mechanisms by which the compression of trachea can be relieved (Figure 5). (I) Excision of the upper sternum and costal cartilages, which are the main structures that lead to the reduction of mediastinal space and compression of the mediastinum. The thickness of the sternum is $\sim 1.5-2.0 \mathrm{~cm}$. If the sternum can be removed and the repair material is placed on the outer surface of the remaining bony structures, the mediastinum will increase by the corresponding width, which is extremely critical for relieving the tracheal compression; (II) the digitally reconstructed material itself is convex, so when it is placed on the outer surface of the bony structure it arches forward, which increases the mediastinal space and further reduces the compression of the trachea; (III) suspension of ascending aorta. The aorta itself has a certain degree of tension and even if the mediastinal space is enlarged, the aorta may still exert a certain pressure on the trachea. In order to completely eliminate this pressure, we suspended the ascending aorta, thus directly eliminating the tracheal pressure. In addition, excision of the enlarged thymus is further enlarged the mediastinal space.

Through these operations, the patient's tracheal compression was completely relieved, the airway resistance was reduced to the normal level, and the symptoms disappeared completely, which proved the effectiveness of this method.

During the operation, we used digitally produced materials to reconstruct the chest wall. Production of the material is similar to the so-called $3 \mathrm{D}$ printing, but the final processing technology of the material is different $(13,14)$. 3D printing materials are printed one at a time by 3D printer (15), whereas digital materials are processed by central navigation computer machine tools. Our experience is that the processing of digital materials is highly controllable and can be specially designed according to the needs of surgery, whereas it is difficult to achieve this goal for 3D printing materials $(15,16)$. Compared with 3D printed materials, digital materials can be personalized, which is more convenient for operation.

For example, the first rib is closely combined with the clavicle. Considering the possibility of subclavian vascular and nerve injuries, we expected that the first costal cartilage would not be reconstructed during the operation. In the process of designing the digital materials, the joint between the first rib and sternum body was designed as a detachable structure. If the first costal cartilage did not need to be reconstructed, the first rib in the digital material can be removed at any time. During our operation, we found that the first rib was hidden, and dense tissue was connected with the clavicle. If resection and reconstruction were performed, it could be dangerous and not essential for increasing mediastinal space, so we finally decided not to reconstruct the first costal cartilage. Therefore, the first rib in the digital material was redundant, so it was removed and finally satisfactory results were obtained. This type of design is quite flexible, which meets the needs of the surgery to the maximum extent, and thus embodies the distinctive feature of personalized design of digital materials.

A similar report (17) was reported this year, but the method is too cumbersome and the postoperative complications are troublesome. They replace and reconstruct the proximal aorta and brachiocephalic artery. Infectious subcutaneous hematoma appeared on the 7 th day after operation, so the patient underwent secondary surgical debridement, negative pressure drainage and the third surgical suture. Our operation is simple and direct, does not destroy too many important tissues, but only reconstructs the chest wall and lifts the aortic arch that compresses the bronchus. There are no postoperative complications and the patient recovers well. The limitation of this study is the single case and the short follow-up time.

In conclusion, increasing the distance between the anterior chest wall and thoracic vertebrae by chest wall reconstruction technology can effectively increase the accommodation space of the mediastinum, reduce the pressure on important structures, and enable SBS to be fundamentally treated. During the operation, the use of digital materials for chest wall reconstruction has many advantages that make the technology a better choice than $3 \mathrm{D}$ printing materials.

\section{Acknowledgments}

Funding: This study was supported by a grant from 
Guangdong Second Provincial People's Hospital (3DA2020003).

\section{Footnote}

Reporting Checklist: The authors have completed the CARE reporting checklist. Available at https://dx.doi. org/10.21037/atm-21-3976

Conflicts of Interest: All authors have completed the ICMJE uniform disclosure form (available at https://dx.doi. org/10.21037/atm-21-3976). All authors reported that this study was supported by a grant from Guangdong Second Provincial People's Hospital (3D-A2020003). The authors have no other conflicts of interest to declare.

Ethical Statement: The authors are accountable for all aspects of the work in ensuring that questions related to the accuracy or integrity of any part of the work are appropriately investigated and resolved. All procedures performed in studies involving human participants were in accordance with the ethical standards of the institutional and/or national research committee(s) and with the Helsinki Declaration (as revised in 2013). Written informed consent was obtained from the patient for publication of this case report and accompanying images. A copy of the written consent is available for review by the editorial office of this journal.

Open Access Statement: This is an Open Access article distributed in accordance with the Creative Commons Attribution-NonCommercial-NoDerivs 4.0 International License (CC BY-NC-ND 4.0), which permits the noncommercial replication and distribution of the article with the strict proviso that no changes or edits are made and the original work is properly cited (including links to both the formal publication through the relevant DOI and the license). See: https://creativecommons.org/licenses/by-nc-nd/4.0/.

\section{References}

1. Rawlings MS: The "straight back" syndrome, a new cause of pseudoheart disease. Am J Cardiol 1960;5:333-8.

2. Betz JW, Oakley PA, Harrison DE. Relief of exertional dyspnea and spinal pains by increasing the thoracic kyphosis in straight back syndrome (thoracic hypokyphosis) using $\mathrm{CBP} \circledast$ methods: a case report with longterm follow-up. J Phys Ther Sci 2018;30:185-9.
3. Hasegawa K, Takaya T, Mori S, et al. Compression of the Right Ventricular Outflow Tract due to Straight Back Syndrome Clarified by Low-dose Dual-source Computed Tomography. Intern Med 2016;55:3279-83.

4. Matsumoto Y, Nitta M, Nakashima R, et al. A mechanism of a cardiac murmur with respiratory variation in a patient with straight back syndrome. J Cardiol Cases 2020;22:230-3.

5. Chen WW, Chan FL, Wong PH, et al. Familial occurrence of mitral valve prolapse: is this related to the straight back syndrome? Br Heart J 1983;50:97-100.

6. Almanfi A, Patel SY, Garcia J, et al. Straight Back Syndrome Masquerading as a Mass, with Near-Syncope. Tex Heart Inst J 2016;43:197-8.

7. Gold PM, Albright B, Anani S, et al. Straight Back Syndrome: positive response to spinal manipulation and adjunctive therapy - A case report. J Can Chiropr Assoc 2013;57:143-9.

8. Mitchell JR, Oakley PA, Harrison DE. Nonsurgical correction of straight back syndrome (thoracic hypokyphosis), increased lung capacity and resolution of exertional dyspnea by thoracic hyperkyphosis mirror image ${ }^{\circledR}$ traction: a CBP ${ }^{\circledR}$ case report. J Phys Ther Sci 2017;29:2058-61.

9. Muyor JM, López-Miñarro PA, Alacid F. Spinal posture of thoracic and lumbar spine and pelvic tilt in highly trained cyclists. J Sports Sci Med 2011;10:355-61.

10. Wang WL, Chen CM, Long WG, et al. Wang procedure: novel minimally invasive procedure for pectus excavatum children with low age. Case Reports and Images in Surgery 2018;1:1-2.

11. Wang W, Chen C. Minimally Invasive Repair of Pectus Carinatum: Bar Number and Technique. Ann Thorac Surg 2018;106:1265-6.

12. Wang W. Modified Ravitch Procedure or Nuss Procedure? Ann Thorac Surg 2018;106:1261-2.

13. Simal I, García-Casillas MA, Cerdá JA, et al. ThreeDimensional Custom-Made Titanium Ribs for Reconstruction of a Large Chest Wall Defect. European J Pediatr Surg Rep 2016;4:26-30.

14. Wu Y, Chen N, Xu Z, et al. Application of 3D printing technology to thoracic wall tumor resection and thoracic wall reconstruction. J Thorac Dis 2018;10:6880-90.

15. Vannucci J, Scarnecchia E, Potenza R, et al. Dynamic titanium prosthesis based on 3D-printed replica for chest wall resection and reconstruction. Transl Lung Cancer Res 2020;9:2027-32.

16. Wang $W$, Liang $Z$, Yang $S$, et al. Three-dimensional (3D)- 
Page 10 of 10

printed titanium sternum replacement: A case report.

Thorac Cancer 2020;11:3375-8.

17. Schmid S, David S, Johannes K, et al. Reconstruction
Liu et al. Diagnosis, symptoms, surgical treatment and outcome of SBS

of the mediastinum and tracheopexy for tracheomalacia in straight back syndrome. Ann Thorac Surg 2021;112:e41-4.

Cite this article as: Liu Y, Wang W, Long W, Cai B, Chen C, Wang W, Guan S, Luo J, Chen K. Chest wall reconstruction with digitally designed materials for straight back syndrome with tracheal stenosis: a case report. Ann Transl Med 2021;9(16):1357. doi: 10.21037/atm-21-3976 